\title{
Lesiones subprotésicas en pacientes portadores de prótesis completa.
}

\author{
Sub-prosthetic injuries in patients carrying total denture
}

\begin{abstract}
Resumen
El propósito de este estudio fue conocer las lesiones que se presentan en los tejidos blandos de soporte en pacientes portadores de prótesis total que acudieron a la clínica odontológica de la Universidad Nacional Mayor de San Marcos (UNMSM) en el año 2012. Se evaluaron 40 pacientes entre 53 y 86 ańos de edad, previa calibración con la finalidad de determinar los cambios que experimenta la mucosa de soporte. A todos se les realizó un frotis citológico. Cada muestra fue coloreada con la técnica de Papanicolaou e interpretada según el sistema Bethesda. Los resultados indicaron que un $95 \%$ presentó lesiones de la mucosa, del cual el $10 \%$ presentó células displásicas, $7.5 \%$ leucoplasias, $55 \%$ estomatitis, $17.5 \%$ hiperplasia y $5 \%$ úlcera. El $90 \%$ de las prótesis presentaron inestabilidad. No se observó diferencias significativas entre los diferentes esquemas oclusales. Por tanto se concluye que la gran mayoría de pacientes que presentan prótesis inestables han desarrollado lesiones sub-protésicas; por lo que se considera necesario el control periódico de las prótesis con un tiempo de uso mayor a un ańo por parte del especialista para evitar lesiones de mayor malignidad.
\end{abstract}

Palabras clave: Prótesis dental, patología bucal, candidiasis bucal.

\section{Abstract}

The purpose of this study was to determine the injuries that occur in soft tissue support in patients carrying total denture attended at dental clinic of San Marcos University in 2012. There were 40 evaluated patients between 53 and 86 years old, previous calibration. In order to determine the changes into the mucosa of support, all patients underwent a cytological smear. Each sample was stained with the Papanicolaou technique and performed with Bethesda system. The results indicated that $95 \%$ had mucosal lesions, of which $10 \%$ had dysplasia cells, 7.5\% leukoplakia stomatitis $55 \%, 17.5 \%$ hyperplasia, $5 \%$ ulcer. The $90 \%$ of the prostheses presented instability. No significant difference was observed in the different occlusal schemes. Therefore, we concluded that the majority of patients with unstable prosthesis have developed sub-prosthetic injuries; therefore we emphasize that the prosthesis over one year of use should have a regular monitoring by the specialist, avoiding malignant lesions.

Keywords: Dental prostheses, oral pathology, oral thrush.

\section{Introducción}

La pérdida total de los dientes genera cambios en la cavidad bucal, tales como la disminución del reborde alveolar, así como la pérdida del funcionalismo masticatorio. ${ }^{1-2}$

La pérdida de los dientes representa un serio problema para la calidad de vida del individuo, pues los mismos además de ser un órgano funcional de la masticación, constituye un componente fundamental de la apariencia individual, el hablar y la comunicación interpersonal.3 La pérdida total de los dientes puede ser resuelta a través de la rehabilitación protésica. La prótesis total convencional ha sido el tratamiento rehabilitador de elección; éstas dentaduras, variables en su diseńo, y tipo de soporte permite restituirle al paciente su funcionalismo masticatorio y la estética facial. ${ }^{4-5}$
La mucosa bucal está constituida por un epitelio escamoso estratificado, para mantener su estructura este epitelio utiliza la renovación celular, producida por la división mitótica, que migran hacia la superficie para reemplazar a aquellos que se descaman. La actividad mitótica puede ser afectada por factores como la presión de la prótesis mal adaptada por ejemplo. ${ }^{5-6}$

La adecuada confección de las dentaduras totales proporcionan una buena retención y estabilidad, para ser mejor tolerado por los tejidos de soporte. Es sabido que los pacientes portadores de dentaduras completas son afectados por la ruptura de este equilibrio de fuerzas; con frecuencia pasa a ser una injuria a la mucosa bucal y de esta manera produce un trauma e inflamación, las dentaduras mucosoportadas están apoyadas en el tejido epitelial que es un tejido ortoqueratenizante con un estrato córneo

\section{Ernesto Vilchez Salazar ${ }^{1}$, Romel Watanabe Velásquez ${ }^{1}$, Soledad Reyes Soto², Ernesto Vilchez Quintana ${ }^{3}$, Carol Torres Roque ${ }^{3}$, Marisabel Vilchez Quintana ${ }^{4}$}

1 Profesor de Rehabilitación Oral de la Facultad de Odontología de la UNMSM.

2 Profesora de Ciencias Básicas Facultad de Odontología de la UNMSM.

3 Bachiller en Odontología de la UNMSM.

4 Alumna de odontología de la UPCH.

Correspondencia:

Mg. Esp. Ernesto Vilchez Salazar.

Av. Germán Amézaga s/n, Ciudad Universitaria Facultad de Odontología de la UNMSM. Teléfono: 993114396

E-mail: ernyrex@hotmail.com

Fecha de recepción: 08 de abril

Fecha de aceptación: 14 de junio

bien desarrollado, sobre las bases de éstas, recaen fuerzas verticales y horizontales. $^{7-8}$

Feltrin, P. realizó un estudio clínico de la mucosa bucal en 80 pacientes portadores de prótesis total encontrando como resultados que el $85 \%$ de pacientes portadores de prótesis total, presentan lesiones inflamatorias en la mucosa de soporte. ${ }^{9}$

Salas, S. realizó una investigación en 130 pacientes portadores de prótesis total removible que acudieron al Hospital Central de la Policial Nacional del Perú, encontrándose como resultado, que el 90\% de pacientes portadores de prótesis totales, presentaron lesiones inflamatorias en los tejidos de soporte. ${ }^{1}$

En países desarrollados el edentulismo está en franca disminución y los tratamientos rehabilitadores se fundamentan en prótesis implanto-soportadas, en 
nuestro país la realidad es totalmente diferente. Las condiciones socioeconómicas de la población condicionan a que la prótesis total sea el tratamiento de elección. El propósito de este estudio fue conocer las lesiones que se presentan en los tejidos blandos de soporte en pacientes portadores de prótesis total que acudieron a la clínica odontológica de la UNMSM en el año 2012

\section{Material y métodos}

Es un estudio clínico analítico, en donde se tomó como muestra 40 pacientes portadores de prótesis completa que acudieron a la clínica odontológica de la Facultad de Odontología de la Universidad Nacional Mayor de San Marcos en el año 2012, seleccionados según el criterio de inclusión.

Se realizó un raspado citológico en los tejidos de soporte del paladar duro de cada paciente utilizando un isopo y una lámina portaobjeto, conservados en alcohol metílico para luego ser coloreados mediante la técnica de Papanicolaou, e interpretados según el sistema Bethesda en microscopio óptico a (40x). Las observaciones fueron registradas con microfotografías. (Fig. 1 y Fig. 3)

Los datos obtenidos fueron consignados en una ficha clínica de recolección de datos, confeccionada y validada para los propósitos de la investigación.

Paralelo a ello se analizaron los cambios morfológicos de los tejidos de soporte de las prótesis totales y se encontraron los diferentes tipos de lesiones.

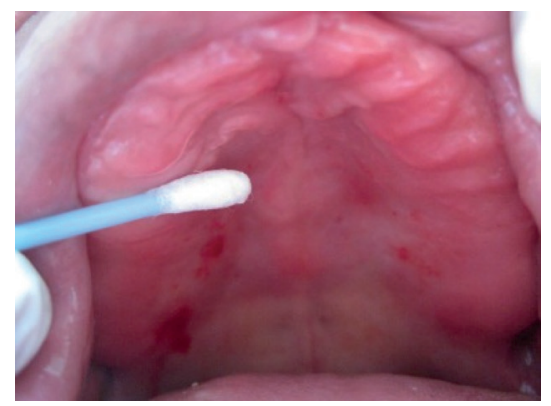

Fig. 1. Mucosa palatina con presencia de estomatitis.

\section{Resultados}

En los 40 frotices citológicos que se realizaron a los pacientes entre 53 a 86 años de edad, se encontró que el 95\% presentó lesiones de la mucosa.

Del total de pacientes, $7.5 \%$ presentó lucoplasias; $55 \%$ estomatitis, $17.5 \%$ hiperplasias; $5 \%$ úlcera y $10 \%$ displasias. (Fig. 2)

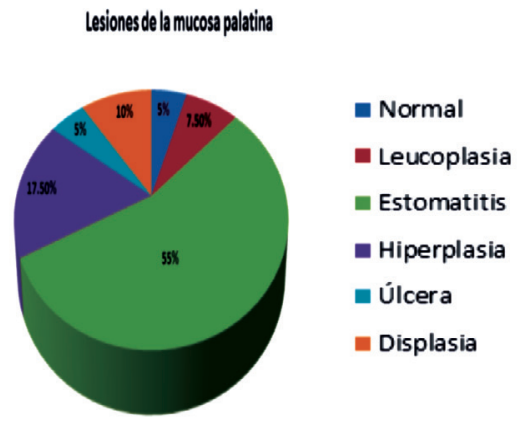

Fig. 2. Se observó mayor presencia de estomatitis en la mucosa palatina.

De los pacientes que presentaron células displásicas, se observó una mayor prevalencia en aquellos que presentaron un mayor tiempo de uso. Igualmente se observó que el mayor porcentaje de displasias se encontró en pacientes mayores a 70 ańos. Sin embargo no se encontró diferencia significativa con el tipo de esquema oclusal utilizado en la confección de la prótesis.

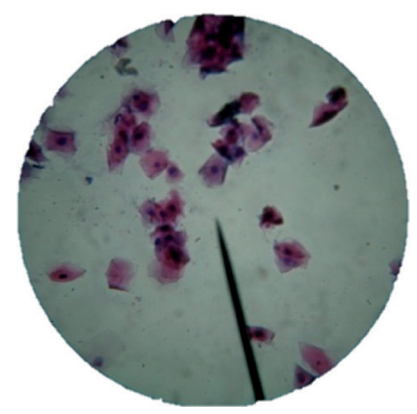

Fig. 3. Microfotografía 40x. Células con displasia leve de la mucosa palatina.

De los pacientes que presentaron estomatitis se observó un $2.5 \%$ de 50 a 60 ańos de edad, un $22.5 \%$ de 61 a 70 años de edad y un 30\% mayores de 70 años, esto indica que los pacientes con mayor edad tienen la mucosa más susceptible a cualquier injuria.

Los pacientes con más de 70 años de edad presentaron mayor prevalencia de lesiones $(62.5 \%)$.

\section{Discusión}

En presente trabajo se reportó presencia de lesiones en la mucosa palatina en $95 \%$ de los pacientes portadores de prótesis total con más de un año de uso.

Santiago S. en un estudio de 130 pacientes portadores de prótesis completa que acudieron al Hospital Central de la Policía Nacional del Perú, encontró como resultado que el $90 \%$ de pacientes portadores de prótesis completa presentaron lesiones inflamatorias en los tejidos de soporte. Nuestra investigación coincide, cuando encuentra el 95 $\%$ de la muestra de 40 pacientes. $^{1}$

Bernal B. (1985) al realizar un estudio de la respuesta inflamatoria celular en la mucosa bucal sub protésica de 60 pacientes, encontró un $9.1 \%$ de células displásicas en las lesiones de la mucosa que presentaron un parecido a lo encontrado en nuestra investigación que arrojó un $10 \%$. $^{3}$

La displasia es un hallazgo muy importante, según criterios actuales para evaluar la pre - malignidad de lesiones de la mucosa, estuvo relacionada con la concentración de células inflamatorias intra epiteliales, fenómeno que puede explicarse considerando a la respuesta inflamatoria como posible factor inductor que estimula el epitelio hacia cambios histológicos de mayor agresividad biológica dado por la dentadura inestables. ${ }^{10-11}$

Los resultados obtenidos muestran que el uso de las prótesis completas, donde se encuentran alterados los principios básicos de confección está asociado a cambios significativos en la diferenciación epitelial, por la antigüedad del uso de la prótesis. ${ }^{12-13-14-15}$

También ejerce un rol importante el tiempo de uso de la prótesis, ya que las prótesis totales con mayor tiempo de uso producen mayor injuria a la mucosa de soporte. ${ }^{16}$

\section{Conclusiones}

Este trabajo confirma que el $95 \%$ de pacientes que portan prótesis completa por más de 1 ańo, presentan cambios citológicos en la mucosa de soporte.

Las lesiones producidas por la prótesis completa inestables pueden llegar a una displasia.

El uso de prótesis completa inestable es causante de las lesiones inflamatorias y displasias.

No se observó diferencia en las lesiones que presentaron los pacientes portadores de prótesis completas con esquema oclusal monoplano y poliplano.

\section{Recomendaciones}

Se debe educar al paciente y difundir los beneficios de un control periódico en los pacientes, por lo menos anualmente.

El examen citomorfológico de la mucosa de soporte debería ser realizado en 
forma periódica en los pacientes portadores de prótesis completa.

Pacientes que usan la prótesis completa por más de 5 años, debe cambiarla o realizar un rebase previa evaluación.

\section{Referencias bibliográficas}

1. Salas S. Lesiones subprotésicas de dentaduras removibles. Revista Gaceta Odontológica 2011; 1 (6): 17-20.

2. Watanabe R. Cambio histométrico de la mucosa bucal en pacientes con prótesis total. Tesis de Maestría. Fac. Odontología: UNMSM. Lima. 1998.

3. Bernal, B; Moreira, D. La respuesta inflamatoria celular en la estomatitis subprotésica. Rev. Cubana de Estomatología, La Habana, Cuba 1985; 22(2): 161-167.

4. Ordie K. Citology-Its value en the diagnosis of cancer. The dental clics on North America, Virginia EE.UU Oct. 2002; 151 (4): 165190.

5. Tortamano N. Estudio da superficie interna da protesis total en mi- croscopia electrónica de barreduras e da mucosa de soporte a través de exame citológico, histopatológico e inmunohistoquimico. Sao PauloBrasil 2002; 49(1): 27-32.

6. Harinasutas. S. Keratinization of palatal mucosa beneath metalbased removable partial dentures In the journal of dentistry, LondresInglaterra. 2004; 20(3): 152-155.

7. Jones A. The cytobrush cell collector In oral cytology. In the oral cytology in the oral surgery, Oral medicine, Oral Patology, Florida EE.UU 2001; 97(1): 101-104.

8. Nakashima K. An experimental study on histopatological changes in the tissue covered with denture base without oclusal presure. In the J. Oral Rehability Japon 2000; 21(3): 263-272.

9. Feltrin. P. Estudio clínico comparativo de la mucosa bucal de pacientes portadores y no portadores de prótesis total Sao Paulo - Brasil 1987; 12(4): 130-138.

10. Jennings, K, Mac Donald D. Histological microbiological and haematological investigatons in denture-induced stomatitis. In the journal of Dentistry. Londres - Inglaterra 2001; 58(2): 102-106.

11. Winkler S. Prostodoncia Total. $2^{\circ}$ ed. México: Editorial Interamericana. 1990; 4 (1): 401-461.

12. Boucher C. Prostodoncia Total. $1^{\circ}$ ed. México: Editorial Interamericana. 1998; 3(2):230-238.

13. Kawabe A. Dentaduras Totales $1^{\circ}$ ed. Japón. Interamericana. 2001; 80-87.

14. Alfred H. Prótesis Total y Sobredentaduras. $1^{\circ}$ ed. Espańa. Interamericana. 2001; 144-118.

15. Bernald B; Moreira D. La respuesta inflamatoria celular en la estomatitis subprotésica. Rev. Cubana de Estomatología; 1995; 15(2): 161-167.

16. Plasencia J. Prótesis Completa. $1^{\circ}$ ed. Espańa: Interamericana. 2001; 65-71.

17. Koeck B. Prótesis Completas. $1^{\circ}$ ed. Espańa: Interamericana. 2001; 81-85. 